\title{
A high-frequency based asymptotic solution for surface fields on a source-excited sphere with an impedance boundary condition
}

\author{
B. Alisan ${ }^{1,2}$ and V. B. Ertürk ${ }^{1}$
}

Received 27 January 2010; revised 4 June 2010; accepted 17 June 2010; published 5 October 2010.

[1] A high-frequency asymptotic solution based on the Uniform Geometrical Theory of Diffraction (UTD) is proposed for the surface fields excited by a magnetic source located on the surface of a sphere with an impedance boundary condition. The assumed large parameters, compared to the wavelength, are the radius of the sphere and the distance between the source and observation points along the geodesic path, when both these points are located on the surface of the sphere. Different from the UTD-based solution for a perfect electrically conducting sphere, some higher-order terms and derivatives of Fock type integrals are included as they may become important for certain surface impedance values as well as for certain separations between the source and observation points. This work is especially useful in the analysis of mutual coupling between conformal slot/aperture antennas on a thin material coated or partially coated sphere.

Citation: Alisan, B., and V. B. Ertürk (2010), A high-frequency based asymptotic solution for surface fields on a source-excited sphere with an impedance boundary condition, Radio Sci., 45, RS5008, doi:10.1029/2010RS004367.

\section{Introduction}

[2] A significant number of military and commercial applications require slot/aperture type antennas that are conformal to the surface of a perfect electrically conducting (PEC) sphere coated or partially coated with a lossy thin dielectric/magnetic material [Tomasic et al., 2002; Sipus et al., 2008]. Thus, the electromagnetic compatibility $(\mathrm{EMC})$ and the electromagnetic interference (EMI) between these antennas become important, and their prediction requires an accurate, and if possible efficient, analysis of mutual coupling between the antennas and hence, surface fields excited by these antennas. However, such an analysis becomes a challenging task when the radius of the sphere and the distance between the antennas along the geodesic path are large in terms of the wavelength. A possible remedy for this challenging task is to approximate the boundary conditions on aforementioned spherical surfaces by an impedance boundary condition [Penney et al., 1996; Rojas and Al-hekail, 1989;

\footnotetext{
${ }^{1}$ Department of Electrical and Electronics Engineering, Bilkent University, Ankara, Turkey.

${ }^{2}$ Aselsan Electronics Inc., Ankara, Turkey.

Copyright 2010 by the American Geophysical Union. 0048-6604/10/2010RS004367
}

Senior and Volakis, 1995], and to perform the analysis using a high-frequency based asymptotic solution that, in general, contain a Fock type integral representation [Fock, 1965].

[3] Several high-frequency based asymptotic solutions for the radio wave propagation around the earth that model the earth by a spherical impedance surface have been presented [Fock, 1945, 1965; Wait, 1960, 1962, 1965, 1967; Spies and Wait, 1966, 1967; Hill and Wait, 1980, 1981; King and Wait, 1976], and attracted significant attention. Among them, Wait [1960] discusses the surface waves excited by a vertical dipole and their propagation on a sphere where the spherical surface exhibits an inductive reactance. In his solution, the electric field is expressed as the radiation field of the dipole if it were placed on the surface of a PEC plane multiplied by an attenuation factor (ground wave attenuation factor) that takes the curvature effects into account and possess a Fock type integral representation. Spies and Wait [1966] discuss the calculation of this ground wave attenuation factor at low frequencies. They use both residue series and power series based on the distance of the observation point from the source. In the work of Spies and Wait [1967], analytical and numerical procedures are described for the evaluation of some Fock type integral functions that appear in a method presented by Wait [1967] to compute the tangential magnetic field on the surface of a smooth inhomogeneous earth excited by a plane wave. 


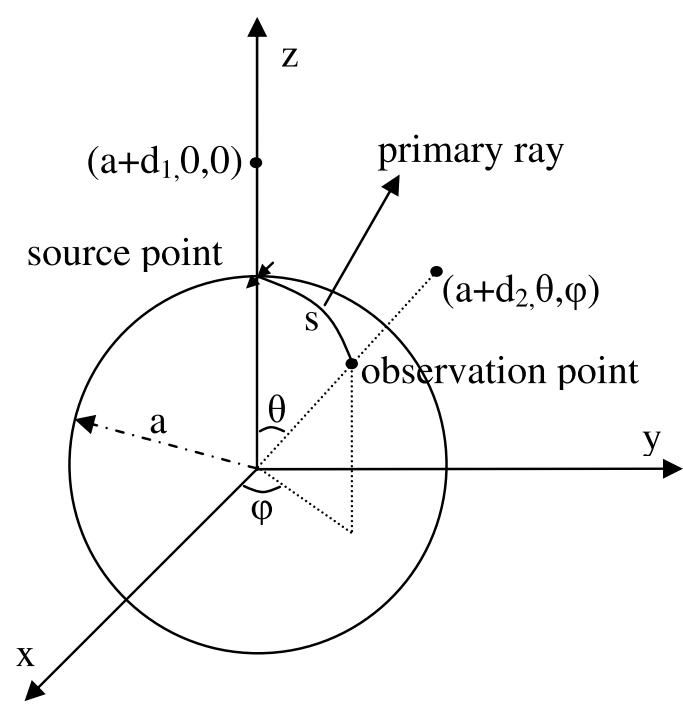

Figure 1. Geometry of a sphere with a radius $a$.

Then, Hill and Wait [1980] generalize the computation of the ground wave attenuation function for a spherical earth with an arbitrary surface impedance, where ground waves are excited by a vertical electric dipole located at the surface of the earth. Their attenuation function is represented in terms of a Fock type integral, and is in general computed using a residue series approach. However, when the argument of the attenuation function is small (i.e., small curvature case), the attenuation function is computed preferably using either its power series representation given by Bremmer [1958] and Wait [1956, 1958], or its small curvature expansion [Wait, 1956; Bremmer, 1958] based on the complementary error function. More references on the subject of ground wave propagation, including the early work, can be found in the work of Wait [1998].

[4] However, the aforementioned solutions are in general valid far from the source location. Therefore, a different high-frequency based asymptotic analysis from that used traditionally in the ground wave propagation problems is developed in this paper. Our solution is a uniform geometrical theory of diffraction (UTD) [Kouyoumjian and Pathak, 1974] based representation of the surface fields excited by a magnetic current located on the surface of a sphere that has a uniform surface impedance, $Z_{s}$ with a positive real part. The radius of the sphere and the length of the geodesic path between the source and observation points, when both are located on the surface of the sphere, are assumed to be large compared to the wavelength. Unlike the UTD-based solution for a PEC sphere developed by Pathak and Wang [1978], some higher-order terms and derivatives of Fock type integrals are included as they may become important for certain impedance values. It is shown that when $Z_{s} \rightarrow 0$, our UTD-based solution recovers to that of PEC case developed by Pathak and Wang [1978] with higher-order terms and derivatives of the corresponding Fock type integrals. Furthermore, the methodology developed by Pathak and Wang [1978] to correct the surface fields at the caustic of the PEC sphere is extended to the impedance sphere case. It should be noted that, together with the UTD-based solution for a circular cylinder with impedance boundary condition (IBC) [Tokgöz and Marhefka, 2006; Alisan et al., 2006], the solution presented in this paper may form a basis toward the development of UTD-based asymptotic solutions valid for arbitrary smooth convex surfaces with an IBC that can model thin material coated/ partially material coated surfaces [Pathak and Wang, 1978, 1981].

[5] The organization of this paper is as follows: Section 2 presents the formulation of the UTD-based solution for the surface fields on an impedance sphere excited by a magnetic current located on the surface of the sphere. In the course of obtaining the high-frequency representations for the surface fields, first a method similar to that developed by Fock [1965] is followed to obtain the necessary potentials without any assumption or approximation, and then UTD-based high-frequency solution is obtained in a similar manner to that developed by Pathak and Wang [1978]. Caustic corrections, limiting situations (i.e., $Z_{s} \rightarrow 0$ ) and numerical evaluation of Fock type integrals are also provided in this section. Numerical results are presented in section 3 , followed by a brief conclusion. An $e^{j \omega t}$ time convention is assumed and suppressed through out this paper, where $\omega=2 \pi f$ with $f$ being the operating frequency.

\section{Formulation}

[6] A UTD-based solution for the surface magnetic field excited by a tangential magnetic source located on the surface of an electrically large sphere with an IBC is presented in this section. Figure 1 illustrates the geometry of interest, where a sphere with a radius of $a$ has a uniform surface impedance $Z_{s}$ that has a nonnegative real part. A magnetic source is defined as $\bar{M}=\hat{x} p_{m} \delta\left(\bar{r}-\bar{r}^{\prime}\right)$ and is located at the point $\left(r^{\prime}=a, \theta^{\prime}=0, \phi^{\prime}=0\right)$ on the sphere. The tangential magnetic fields $H_{\theta}(\bar{r})$ and $H_{\phi}(\bar{r})$ are calculated at the field point $(r=a, \theta, \phi)$ on the surface of the sphere. The distance between the source and field points along the geodesic path is denoted by $s=a \theta$, which is also indicating the primary ray direction.

\subsection{Derivation of the Surface Fields}

[7] The starting point of the formulation is similar to that of Fock [1965], where a vector potential $\bar{F}_{0}$ due to a source $\bar{M}$ in the absence of the impedance sphere can be 
represented by an infinite sum of spherical wave functions of the form

$$
\begin{gathered}
\bar{F}_{0}=\hat{x} \frac{k p_{m}}{4 \pi j} \sum_{n=0}^{\infty}(2 n+1) h_{n}^{(2)}\left(k r^{\prime}\right) j_{n}(k r) P_{n}(\cos \theta) ; \\
|\bar{r}|<\left|\bar{r}^{\prime}\right|
\end{gathered}
$$

where $j_{n}, h_{n}^{(2)}$ and $P_{n}$ are the usual spherical Bessel, Hankel and Legendre functions [Abramowitz and Stegun, 1964], respectively. Defining a set of potentials, $\psi_{0}^{e} \hat{r}$ and $\psi_{0}^{m} \hat{r}$ associated with $\bar{M}$ in the free space that satisfy

$$
\left(\nabla^{2}+k^{2}\right)\left\{\begin{array}{c}
\psi_{0}^{e} / r \\
\psi_{0}^{m} / r
\end{array}\right\}=0 ; \quad r \neq 0
$$

are related to $\bar{F}_{0}$ via

$$
\begin{gathered}
\sin \phi \frac{\partial F_{0}}{\partial \theta}=\frac{-1}{j k Y_{0}} \Delta^{*}\left(\frac{\psi_{0}^{e}}{r}\right) \\
\cos \phi\left[\frac{\partial}{\partial \theta}\left(\frac{\partial F_{0}}{\partial b}+\frac{F_{0}}{b}\right)\right]=\Delta^{*}\left(\frac{\psi_{0}^{m}}{r}\right)
\end{gathered}
$$

where the operator $\Delta^{*}$ is defined by Fock [1965] as

$$
\Delta^{*}=\frac{1}{\sin \theta} \frac{\partial}{\partial \theta}\left(\sin \theta \frac{\partial}{\partial \theta}\right)+\frac{1}{\sin ^{2} \theta} \frac{\partial^{2}}{\partial \phi^{2}},
$$

and source is initially assumed to be at $\bar{r}^{\prime}=\left(a+d_{1}\right) \hat{z}=b \hat{z}$ as illustrated in Figure 1. In (3), $Y_{0}=1 / Z_{0}$ is the freespace admittance. Substituting (1) into (3) and (4), and using the properties given by [Balanis, 1989]

$$
\begin{gathered}
\hat{J}_{n}(k r)=k r j_{n}(k r) \\
\hat{H}_{n}^{(2)}(k r)=k r h_{n}^{(2)}(k r) \\
\frac{\partial}{\partial \theta} P_{n}(\cos \theta)=P_{n}^{1}(\cos \theta)
\end{gathered}
$$

$\psi_{0}^{e}$ and $\psi_{0}^{m}$ can be expressed as

$$
\begin{aligned}
\psi_{0}^{e}= & \left(\frac{k p_{m}}{4 \pi j}\right)\left(\frac{j Y_{0} \sin \phi}{k b}\right) \sum_{n=1}^{\infty} \frac{2 n+1}{n(n+1)} \\
& \cdot \hat{H}_{n}^{(2)}(k b) \hat{J}_{n}(k r) P_{n}^{1}(\cos \theta) \\
\psi_{0}^{m}= & \left(\frac{k p_{m}}{4 \pi j}\right)\left(\frac{-\cos \phi}{k b}\right) \sum_{n=1}^{\infty} \frac{2 n+1}{n(n+1)} \\
& \cdot \hat{H}_{n}^{(2)^{\prime}}(k b) \hat{J}_{n}(k r) P_{n}^{1}(\cos \theta)
\end{aligned}
$$

where ' denotes the derivative with respect to the argument. The scattered fields due to the presence of the impedance sphere are in similar form to those of incident fields (i.e., in the form of an infinite sum of spherical wave functions) except some complex coefficients to be found from the appropriate boundary conditions. Thus, defining another set of potentials to account for the scattering from the impedance sphere, and superposing them with the free-space potentials defined in (9) and (10), the total potentials are given by

$$
\begin{aligned}
\psi^{e}= & \left(\frac{k p_{m}}{4 \pi j}\right)\left(\frac{j Y_{0} \sin \phi}{k b}\right) \sum_{n=1}^{\infty} \frac{2 n+1}{n(n+1)} \\
& \cdot \hat{H}_{n}^{(2)}(k b)\left(\hat{J}_{n}(k r)+C_{1 n} \hat{H}_{n}^{(2)}(k r)\right) P_{n}^{1}(\cos \theta)
\end{aligned}
$$

$$
\begin{aligned}
\psi^{m}= & \left(\frac{k p_{m}}{4 \pi j}\right)\left(\frac{-\cos \phi}{k b}\right) \sum_{n=1}^{\infty} \frac{2 n+1}{n(n+1)} \\
& \cdot \hat{H}_{n}^{(2)^{\prime}}(k b)\left(\hat{J}_{n}(k r)+C_{2 n} \hat{H}_{n}^{(2)}(k r)\right) P_{n}^{1}(\cos \theta) .
\end{aligned}
$$

In (11) and (12), $C_{1 n}$ and $C_{2 n}$ are complex coefficients to be found by applying the impedance boundary conditions at $r=a$ given by

$$
\left[\begin{array}{c}
E_{\phi} \\
H_{\phi}
\end{array}\right]=\left[\begin{array}{cc}
Z_{s} & 0 \\
0 & -Z_{s}^{-1}
\end{array}\right]\left[\begin{array}{c}
H_{\theta} \\
E_{\theta}
\end{array}\right]
$$

[8] Therefore, calculating $E_{\phi}$ and $H_{\theta}$ from $\psi^{e}$ and $\psi^{m}$ using [Harrington, 1961]

$$
\begin{aligned}
& E_{\phi}=\frac{1}{r} \frac{\partial \psi^{m}}{\partial \theta}+\frac{1}{j k Y_{0} r \sin \theta} \frac{\partial^{2} \psi^{e}}{\partial r \partial \phi} \\
& H_{\theta}=\frac{1}{r \sin \theta} \frac{\partial \psi^{e}}{\partial \phi}+\frac{1}{j k Z_{0} r} \frac{\partial^{2} \psi^{m}}{\partial r \partial \theta},
\end{aligned}
$$

and substituting the results into (13), the complex coefficients $C_{1 n}$ and $C_{2 n}$ are obtained as

$$
\begin{gathered}
C_{1 n}=-\frac{\hat{J}_{n}(k a)+j \Lambda^{-1} \hat{J}_{n}^{\prime}(k a)}{\hat{H}_{n}^{(2)}(k a)+j \Lambda^{-1} \hat{H}_{n}^{(2)^{\prime}}(k a)} \\
C_{2 n}=-\frac{\hat{J}_{n}(k a)+j \Lambda \hat{J}_{n}^{\prime}(k a)}{\hat{H}_{n}^{(2)}(k a)+j \Lambda \hat{H}_{n}^{(2)^{\prime}}(k a)}
\end{gathered}
$$

where $\Lambda$ is the normalized surface impedance and defined as $Z_{s} / Z_{0}$. 


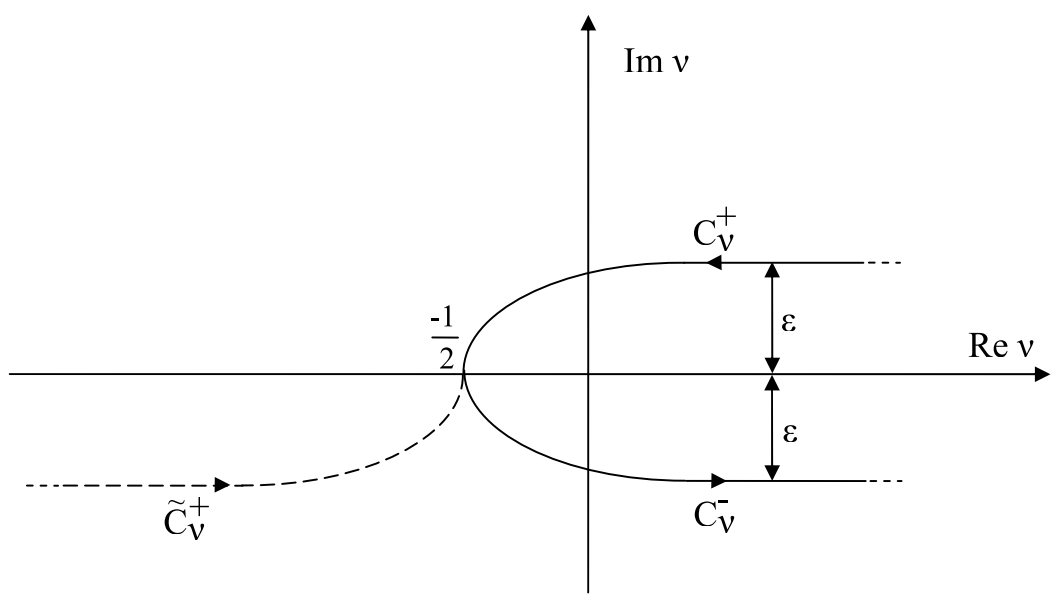

Figure 2. Contour of integration in the complex $\nu$ plane. $C_{\nu}=C_{\nu}^{+}+C_{\nu}^{-}$is the original contour and $\tilde{C}_{\nu}=\tilde{C}_{\nu}^{+}+C_{\nu}^{-}$is the new contour.

[9] Finally, substituting (16) and (17) into (11) and (12), the exact expressions for $\psi^{e}$ and $\psi^{m}$ can be found as

$$
\begin{aligned}
\psi^{e}= & \left(\frac{k p_{m}}{4 \pi j}\right)\left(\frac{j Y_{0} \sin \phi}{k b}\right) \sum_{n=1}^{\infty} \frac{2 n+1}{n(n+1)} \\
& \cdot \hat{H}_{n}^{(2)}(k b) \tilde{A}_{n}(k r) P_{n}^{1}(\cos \theta) \\
\psi^{m}= & \left(\frac{k p_{m}}{4 \pi j}\right)\left(\frac{-\cos \phi}{k b}\right) \sum_{n=1}^{\infty} \frac{2 n+1}{n(n+1)} \\
& \cdot \hat{H}_{n}^{(2)^{\prime}}(k b) \tilde{B}_{n}(k r) P_{n}^{1}(\cos \theta)
\end{aligned}
$$

where

$$
\begin{aligned}
& \tilde{A}_{n}(k r)=\hat{J}_{n}(k r)-\frac{\hat{J}_{n}(k a)+j \Lambda^{-1} \hat{J}_{n}^{\prime}(k a)}{\hat{H}_{n}^{(2)}(k a)+j \Lambda^{-1} \hat{H}_{n}^{(2)^{\prime}}(k a)} \hat{H}_{n}^{(2)}(k r) \\
& \tilde{B}_{n}(k r)=\hat{J}_{n}(k r)-\frac{\hat{J}_{n}(k a)+j \Lambda \hat{J}_{n}^{\prime}(k a)}{\hat{H}_{n}^{(2)}(k a)+j \Lambda \hat{H}_{n}^{(2)^{\prime}}(k a)} \hat{H}_{n}^{(2)}(k r) .
\end{aligned}
$$

[10] At this stage, one can find the exact expression for the field components from the potentials $\psi^{e}$ and $\psi^{m}$ that involve infinite summations, and then find the highfrequency based asymptotic expressions for these field components. However, in this paper we prefer to use an alternative method developed by Pathak and Wang [1978] for PEC cylinder and sphere. Briefly, it is a two-step procedure where the leading term $[O(1 / k s)]$ of the highfrequency based expressions for the potentials $\left(\psi^{e}\right.$ and $\psi^{m}$ in this study) are first developed, and the fields are then obtained by taking the necessary derivatives. However, unlike Pathak and Wang [1978] some higher-order terms and derivatives of Fock type integrals are retained as they may be important for some $Z_{S}$ values for some separations between the source and observation points. Note that recently a similar procedure has been followed by Tokgöz and Marhefka [2006] to find the UTD-based solution for the surface fields on an impedance cylinder.

[11] The first step of the high-frequency development of the surface fields on an impedance sphere is to apply Watson's transformation [Watson, 1918] to convert the very slowly convergent infinite summations in (18) and (19) to a contour integral $C_{\nu}^{ \pm}$, as shown in Figure 2. Thus, the new expression for the potentials are given by

$$
\begin{aligned}
\left\{\begin{array}{c}
\psi^{e} \\
\psi^{m}
\end{array}\right\}= & \frac{k p_{m}}{4 \pi j} \frac{1}{k b}\left\{\begin{array}{c}
j Y_{0} \sin \phi \\
-\cos \phi
\end{array}\right\} \frac{1}{2 \pi j} \oint_{C_{\nu}^{+}+C_{\nu}^{-}} \\
& \cdot d \nu \frac{2 \nu+1}{\nu(\nu+1)} \frac{\pi(-1)^{\nu}}{\sin (\nu \pi)} \\
& \cdot \hat{H}_{\nu}^{(2)}(k b)\left\{\begin{array}{c}
\tilde{A}_{\nu}(k r) \\
\tilde{B}_{\nu}(k r)
\end{array}\right\} P_{\nu}^{1}(\cos \theta) .
\end{aligned}
$$

[12] Then, as suggested by Pathak and Wang [1978], using the relation [Abramowitz and Stegun, 1964]

$$
(-1)^{\nu} P_{\nu}^{1}(\cos \theta)=\nu(\nu+1) P_{\nu}^{-1}(-\cos \theta)
$$


and then replacing $\nu$ by $-\nu-1$ in the integration over $C_{\nu}^{+}$, the potentials are obtained as

$$
\begin{aligned}
\left\{\begin{array}{c}
\psi^{e} \\
\psi^{m}
\end{array}\right\}= & \frac{k p_{m}}{4 \pi j} \frac{1}{k b}\left\{\begin{array}{c}
j Y_{0} \sin \phi \\
-\cos \phi
\end{array}\right\} \frac{1}{2 \pi j} \int_{-\infty-j \xi}^{\infty-j \xi} \\
& \cdot d \nu \frac{\pi}{\sin (\nu \pi)}(2 \nu+1) \\
& \cdot \hat{H}_{\nu}^{(2)}(k b)\left\{\begin{array}{c}
\tilde{A}_{\nu}(k r) \\
\tilde{B}_{\nu}(k r)
\end{array}\right\} P_{\nu}^{-1}(-\cos \theta) .
\end{aligned}
$$

[13] As the next step, the potentials $\psi^{e}$ and $\psi^{m}$ are evaluated at $r=r^{\prime}=a$, and the integration variable is changed from $\nu$ to $\mu$ via $\mu=\nu+1 / 2$. Then, the substitution originally suggested by [Fock, 1945]

$$
\mu=k a+m \tau \quad ; \quad m=\left(\frac{k a}{2}\right)^{1 / 3}
$$

is made, and the series expansion of $\frac{1}{\sin [(\mu-1 / 2) \pi]}$ given by

$$
\frac{1}{\sin [(\mu-1 / 2) \pi]}=2 j e^{-j(\mu-1 / 2) \pi} \sum_{\ell=0}^{\infty} e^{-j(\mu-1 / 2)(2 \pi \ell)}
$$

is employed where only the $\ell=0$ term is retained since $\ell \neq 0$ terms correspond to multiple encirclements around the sphere and are negligible for large $k a$. Finally, replacing the cylindrical Hankel and Bessel functions (i.e., $\left.\hat{J}_{\mu^{-1 / 2}}(k a), \hat{H}_{\mu^{-1 / 2}}^{(2)}(k a)\right)$ along with their derivatives by Fock type Airy functions and their derivatives (provided in Appendix A for the sake of completeness), and approximating the Legendre polynomial $P_{\mu-1 / 2}^{-1}(-\cos \theta)$ by [Abramowitz and Stegun, 1964]

$$
\begin{aligned}
& P_{\mu-1 / 2}^{-1}(-\cos \theta) \sim-\frac{\mu^{-3 / 2}}{2 j} \sqrt{\frac{2}{\pi \sin \theta} e^{-j \pi / 4} e^{j \mu \pi}} \\
& \cdot\left[e^{-j \mu \theta}-j e^{-j \mu(2 \pi-\theta)}\right]
\end{aligned}
$$

$\psi^{e}$ can be obtained as

$$
\begin{gathered}
\psi^{e} \sim\left(\frac{k p_{m}}{4 \pi j}\right)\left(\frac{-j Y_{0} \sin \phi}{k a}\right) \sqrt{\frac{2}{\pi \sin \theta} e^{-j \pi / 4} j m} \int_{-\infty}^{\infty} d \mu \mu^{-1 / 2} \\
\cdot\left[\frac{1}{R_{w}(\tau)+j m \Lambda}\right]\left[e^{-j \mu \theta}-j e^{-j \mu(2 \pi-\theta)}\right]
\end{gathered}
$$

where

$$
R_{w}(\tau)=\frac{W_{2}^{\prime}(\tau)}{W_{2}(\tau)}
$$

[14] Note that $\psi^{e}$ can be written as $\psi_{+}^{e}$ and $\psi_{-}^{e}$ where $\psi_{+}^{e}$ is associated with $e^{-j \mu \theta}$ term; and $\psi_{-}^{e}$ is associated with $e^{-j \mu(2 \pi-\theta)}$ term [Pathak and Wang, 1978]. Then, using the definitions made by [Pathak and Wang, 1978]:

$$
\begin{gathered}
\xi^{ \pm}=m \theta^{ \pm}=m\left\{\begin{array}{c}
\theta \\
2 \pi-\theta
\end{array}\right\} \\
s^{ \pm}=a\left\{\begin{array}{c}
\theta \\
2 \pi-\theta
\end{array}\right\}
\end{gathered}
$$

and making use of the following approximation for large $k a$

$$
\int d \mu \mu^{-1 / 2} \sim \frac{m}{\sqrt{k a}} \int d \tau
$$

the final form of $\psi^{e}$ is written in the form of $\psi_{ \pm}^{e}$ as

$$
\psi_{ \pm}^{e} \sim \pm \frac{j p_{m}}{k} V_{z}\left(\xi^{ \pm}\right) \sin \phi D^{ \pm} G\left(k s^{ \pm}\right)
$$

where

$$
\begin{gathered}
G\left(k s^{ \pm}\right)=\frac{k^{2} Y_{0}}{2 \pi j} \frac{e^{-j k s^{ \pm}}}{k s^{ \pm}} \\
D^{ \pm}=\sqrt{\frac{\theta^{ \pm}}{\sin \theta^{ \pm}}} \\
V_{z}\left(\xi^{ \pm}\right)=\sqrt{\frac{j \xi^{ \pm}}{4 \pi}} \int_{-\infty}^{\infty} \frac{1}{R_{w}-q_{e}} e^{-j \xi^{ \pm} \tau} d \tau
\end{gathered}
$$

with $q_{e}=-j m \Lambda$. Notice that (33)-(36) are exactly in the same form as that of the PEC case except the integrand of (36). Also note that in (33)-(36), (+) corresponds to the field propagation along the geodesic ray path corresponding to $s^{+}=a \theta^{+}$whereas $(-)$corresponds to the field propagation along the same geodesic ray path but in a direction opposite to $s^{+}$corresponding to $s^{-}=a \theta^{-}=a\left(2 \pi-\theta^{+}\right)$.

[15] Because the tangential magnetic field components $H_{\theta}$ and $H_{\phi}$ contain the derivative of $\psi^{m}$ with respect to $r$, as seen in (15) and

$$
H_{\phi}=-\frac{1}{r} \frac{\partial \psi_{e}}{\partial \theta}+\frac{1}{j k Z_{0} r \sin \theta} \frac{\partial^{2} \psi_{m}}{\partial r \partial \phi}
$$

the Fock substitution is employed as

$$
\begin{aligned}
\mu & =k a+m \tau=k\left(r-d_{2}\right)+m \tau=k r+m\left(\tau-m^{-1} k d_{2}\right) \\
& =k r+m\left(\tau-y_{2}\right),
\end{aligned}
$$

and the evaluation at $r=a$ (i.e., $d_{2}=0$ ) is performed after performing the derivatives with respect to $r$. Thus, 
performing the same definitions and approximation as done for $\psi^{e}$ case, $\psi^{m}$ is written as

$$
\begin{aligned}
\psi_{ \pm}^{m} \sim & \pm \frac{-p_{m} e^{-j \pi / 4}}{4 k m Y_{0}} \cos \phi D^{ \pm} G\left(k s^{ \pm}\right) \sqrt{\frac{\xi^{ \pm}}{\pi}} \int_{-\infty}^{\infty} d \tau e^{-j \xi^{ \pm} \tau} \\
& \cdot \frac{W_{2}^{\prime}(\tau)}{-m W_{2}(\tau)+j \Lambda W_{2}^{\prime}(\tau)}\left\{W_{1}\left(\tau-y_{2}\right)\right. \\
& \cdot\left[-m W_{2}(\tau)+j \Lambda W_{2}^{\prime}(\tau)\right]+W_{2}\left(\tau-y_{2}\right) \\
& \left.\cdot\left[m W_{1}(\tau)-j \Lambda W_{1}^{\prime}(\tau)\right]\right\}
\end{aligned}
$$

before the derivatives are performed and then, as explained in Appendix B, the tangential magnetic field components $H_{\theta}$ and $H_{\phi}$ are found from (15) and (37) as

$$
\begin{aligned}
H_{\theta}^{ \pm}= & \pm p_{m} \cos \phi\left\{\left[\frac{j}{k s^{ \pm}}\left(1-\frac{2 j}{k s^{ \pm}}\right) U_{z}\left(\xi^{ \pm}\right)\right.\right. \\
& \left.+D^{ \pm 2} \frac{j}{k s^{ \pm}} V_{z}\left(\xi^{ \pm}\right)\right] D^{ \pm} G\left(k s^{ \pm}\right) \\
& \left.-G\left(k s^{ \pm}\right) \frac{1}{4 m^{5} \xi^{ \pm}} \frac{\partial}{\partial \theta}\left[D^{ \pm} U_{z}\left(\xi^{ \pm}\right)\right]\right\} \\
= & \pm p_{m} \cos \phi\left[\left(\frac{j}{k s^{ \pm}}\right) \tilde{U}_{z}\left(\xi^{ \pm}\right)-\frac{D^{ \pm}}{2}\left(\frac{j}{k s^{ \pm}}\right)^{2}\right. \\
& \left.\cdot \cos \theta U_{z}\left(\xi^{ \pm}\right)+D^{ \pm} \frac{j}{k s^{ \pm}} V_{z}\left(\xi^{ \pm}\right)\right] D^{ \pm} G\left(k s^{ \pm}\right) \\
H_{\phi}^{ \pm}= & \mp p_{m} \sin \phi\{(41) \\
& \left.\cdot D^{ \pm} G\left(k s^{ \pm}\right)+G\left(k s^{ \pm}\right) \frac{j}{2 m^{3}} \frac{\partial}{\partial \theta}\left[D^{ \pm} V_{z}\left(\xi^{ \pm}\right)\right]\right\} \\
= & \left.\left.\mp p_{m} \sin \phi\left[\tilde{V}_{z}\left(\xi^{ \pm}\right)-\frac{j}{k s^{ \pm}}\right) V_{z}\left(\xi^{ \pm}\right)+j^{2} D^{ \pm} \frac{j}{2} \frac{U_{z}\left(\xi^{ \pm}\right)}{k s^{ \pm}}\right)^{2}\right] \\
& \left.+D^{ \pm 2}\left(\frac{j}{k s^{ \pm}}\right)^{2} U_{z}\left(\xi^{ \pm}\right)\right] D_{z}^{ \pm} G\left(\xi^{ \pm}\right) \\
&
\end{aligned}
$$

where $V_{z}$ and $U_{z}$ are the Fock type integrals given by

$$
\begin{gathered}
V_{z}\left(\xi^{ \pm}\right)=\sqrt{\frac{j \xi^{ \pm}}{4 \pi}} \int_{-\infty}^{\infty} \frac{1}{R_{w}-q_{e}} e^{-j \xi^{ \pm} \tau} d \tau \\
U_{z}\left(\xi^{ \pm}\right)=e^{j 3 \pi / 4} \xi^{ \pm / 2} \frac{1}{\sqrt{\pi}} \int_{-\infty}^{\infty} d \tau e^{-j \xi^{ \pm} \tau} \frac{-R_{w} q_{m}}{\left(R_{w}-q_{m}\right)}
\end{gathered}
$$

$\tilde{V}_{z}$ and $\tilde{U}_{z}$ include the derivatives of $V_{z}$ and $U_{z}$, and are given by

$$
\begin{gathered}
\tilde{V}_{z}\left(\xi^{ \pm}\right)=\sqrt{\frac{j \xi^{ \pm}}{4 \pi}} \int_{-\infty}^{\infty} d \tau e^{-j \xi^{ \pm} \tau} \frac{1}{\left(R_{w}-q_{e}\right)}\left(1+\frac{\tau}{2 m^{2}}\right) \\
\tilde{U}_{z}\left(\xi^{ \pm}\right)=e^{j 3 \pi / 4} \xi^{ \pm 3 / 2} \frac{1}{\sqrt{\pi}} \int_{-\infty}^{\infty} d \tau e^{-j \xi^{ \pm} \tau} \\
\cdot \frac{-R_{w} q_{m}}{\left(R_{w}-q_{m}\right)}\left(1+\frac{\tau}{2 m^{2}}\right)
\end{gathered}
$$

in which $q_{m}=-j m \Lambda^{-1}$, and $q_{e}=-j m \Lambda$ (as given before). Details of obtaining (41) from (40) and (43) from (42) are also given in Appendix B.

[16] Note that in all practical applications two rays (corresponding to $\ell=0$ in (26)) traveling in opposite directions around the impedance sphere yield enough accuracy for relatively large spheres. Also note that the Fock type integrals and the surface field expressions are in similar form to those provided for impedance cylinder by Tokgöz and Marhefka [2006]. Thus, together they can form a basis toward the development of UTD-based solutions for arbitrary smooth convex surfaces with an IBC.

\subsection{Caustic Corrections}

[17] When the field point on the spherical surface is at $\theta=\pi$, it forms a caustic for the surface fields and the tangential magnetic field expressions developed in section 2.1 are not valid due to the $D^{ \pm}$expression (when $\theta \rightarrow \pi, D \rightarrow \infty)$. Therefore, the caustic correction methodology followed in this paper is similar to that performed for PEC sphere problem by Pathak and Wang [1978]. Briefly, in (40)-(43) the expressions have either $D G(k s)$ or $D^{3} G(k s)$ type combinations, and are replaced by the following approximate expressions provided by Pathak and Wang [1978]:

$$
\begin{aligned}
& D^{+} G\left(k s^{+}\right)+D^{-} G\left(k s^{-}\right) \\
& \quad \approx \frac{k^{2} Y_{0}}{2 \pi j}\left[\pi m^{3 / 2} e^{-j 2 \pi m^{3}} J_{0}\left(2 m^{3}(\pi-\theta)\right)\right] \frac{2 e^{j \pi / 4}}{k s^{+}}
\end{aligned}
$$

$$
\begin{aligned}
& {\left[D^{+}\right]^{3} G\left(k s^{+}\right)+\left[D^{-}\right]^{3} G\left(k s^{-}\right)} \\
& \quad \approx \frac{k^{2} Y_{0}}{2 \pi j}\left[2 \pi^{2} m^{9 / 2} e^{-j 2 \pi m^{3}} \frac{J_{1}\left(2 m^{3}(\pi-\theta)\right)}{2 m^{3}(\pi-\theta)}\right] \frac{2 e^{j 3 \pi / 4}}{k s^{+}}
\end{aligned}
$$

where $J_{0}$ and $J_{1}$ are Bessel functions. 


\subsection{Reduction of UTD-Based Solution to the Limiting Case of a PEC Sphere}

[18] When $Z_{s} \rightarrow 0$,

$$
\begin{aligned}
& \lim _{Z_{s} \rightarrow 0} \frac{1}{\left(R_{w}-q_{e}\right)}=\lim _{Z_{s} \rightarrow 0} \frac{1}{\frac{W_{2}^{\prime}(\tau)}{W_{2}(\tau)}+j m \frac{Z_{s}}{Z_{0}}}=\frac{W_{2}(\tau)}{W_{2}^{\prime}(\tau)} \\
& \lim _{Z_{s} \rightarrow 0} \frac{-R_{w} q_{m}}{\left(R_{w}-q_{m}\right)}=\lim _{Z_{s} \rightarrow 0} \frac{\frac{W_{2}^{\prime}(\tau)}{W_{2}(\tau)} j m \frac{Z_{0}}{Z_{s}}}{\frac{W_{2}^{\prime}(\tau)}{W_{2}(\tau)}+j m \frac{Z_{0}}{Z_{s}}}=\frac{W_{2}^{\prime}(\tau)}{W_{2}(\tau)} .
\end{aligned}
$$

Therefore, the Fock type integrals given by (44) and (45) reduce to

$$
\begin{gathered}
\lim _{Z_{s} \rightarrow 0} V_{z}=V=\sqrt{\frac{j \xi}{4 \pi}} \int_{-\infty}^{\infty} d \tau e^{-j \xi \tau} \frac{W_{2}(\tau)}{W_{2}^{\prime}(\tau)} \\
\lim _{Z_{s} \rightarrow 0} U_{z}=U=e^{j 3 \pi / 4} \xi^{3 / 2} \frac{1}{\sqrt{\pi}} \int_{-\infty}^{\infty} d \tau e^{-j \xi \tau} \frac{W_{2}^{\prime}(\tau)}{W_{2}(\tau)} .
\end{gathered}
$$

which are the Fock type functions given by Pathak and Wang [1978] for the PEC sphere problem. Thus, the final expressions of $H_{\theta}$ and $H_{\phi}$ given by (40)-(43) can be obtained in the limit as $Z_{s} \rightarrow 0$ as

$$
\begin{aligned}
H_{\theta}^{ \pm}= & \pm p_{m} \cos \phi\left\{\left[\frac{j}{k s^{ \pm}}\left(1-\frac{2 j}{k s^{ \pm}}\right) U\left(\xi^{ \pm}\right)\right.\right. \\
& \left.+D^{ \pm} \frac{j}{k s^{ \pm}} V\left(\xi^{ \pm}\right)\right] D^{ \pm} G\left(k s^{ \pm}\right) \\
& \left.-G\left(k s^{ \pm}\right) \frac{1}{4 m^{5} \xi^{ \pm}} \frac{\partial}{\partial \theta}\left[D^{ \pm} U\left(\xi^{ \pm}\right)\right]\right\} \\
H_{\phi}^{ \pm}= & \mp p_{m} \sin \phi\left\{\left[\left(1-\frac{j}{k s^{ \pm}}\right) V\left(\xi^{ \pm}\right)+j^{2} D^{ \pm 2} \frac{U\left(\xi^{ \pm}\right)}{\left(k s^{ \pm}\right)^{2}}\right]\right. \\
& \left.\cdot D^{ \pm} G\left(k s^{ \pm}\right)+G\left(k s^{ \pm}\right) \frac{j}{2 m^{3}} \frac{\partial}{\partial \theta}\left[D^{ \pm} V\left(\xi^{ \pm}\right)\right]\right\} . \quad(55)
\end{aligned}
$$

When (54) and (55) are compared with the UTD-based solution for a PEC sphere developed by Pathak and Wang [1978], the third terms in both (54) and (55) (i.e., the terms that contain the derivative with respect to $\theta$ ) are extra, and include some higher-order terms and derivatives of the Fock type integrals, $U$ and $V$. These extra terms were neglected by Pathak and Wang [1978].

\subsection{Computation of Fock Type Integrals}

[19] Computation of the Fock type integrals are performed in two ways and the same accuracy is obtained in both cases. The first approach is to invoke Cauchy's residue theorem. Briefly, the pole singularities of the integrands are found, and the values of the integrals are obtained by summing the residues corresponding to these poles. Details of this approach for impedance sphere are explained by Spies and Wait [1966]. The second approach is to perform a numerical integration, and based on deforming the integration contour on which the integrands of Fock type integrals are non oscillatory and fast decaying. Briefly, these integrals are split into three integrals ranging from $(-\infty, 0),\left(0, \tau_{\text {big }}\right)$ and $\left(\tau_{\text {big }}, \infty\right)$, where $\tau_{\text {big }}$ is chosen approximately $1.5 \mathrm{ka}$ (or $2 \mathrm{ka}$ ) to ensure all pole singularities including a low-attenuation Elliott mode [Logan and Yee, 1962; Hill and Wait, 1980; Felsen and Naishadham, 1991] are captured. Then, the integration variable $\tau$ is changed to $\tau e^{j 2 \pi / 3}$ for the first integral and to $\left(\tau-\tau_{b i g}\right) e^{j \pi / 3}$ for the third integral, causing the Airy function and its derivative to be non oscillatory and fast decaying (an exponential decay is achieved). Only the second integral remains oscillatory but its integration interval is relatively short. Thus, its numerical computation does not impose a difficulty though most of the CPU time is consumed during its computation. Finally, a simple Gaussian quadrature algorithm is used for the integration along this deformed contour. This approach is successfully implemented to a circular cylinder with an IBC, and accurate results are obtained [Alisan et al., 2006]. However, this approach is preferred when it is not easy to locate the poles of the integrand like in the impedance cylinder, coated cylinder, coated sphere cases. The first approach is more efficient for an impedance sphere.

\section{Numerical Results}

[20] In this section, several numerical results for the surface magnetic field on an electrically large sphere with an impedance boundary condition are given to illustrate the validity and the accuracy of our proposed solution.

[21] The first set of numerical results aims to verify the validity of our solution. Therefore, tangential magnetic field components, $H_{\theta}$ and $H_{\phi}$, are computed for the geodesic path length varying from $0.1 \lambda$ to $31.3 \lambda$ at $f=$ $7 \mathrm{GHz}$ for a fixed azimuthal angle $\left(\phi=45^{\circ}\right)$ on spheres with a radius $5 \lambda$ having different surface impedances. Surface impedances are chosen to be in the form of $Z_{s}=$ $\alpha+j \beta$ or $Z_{s}=\alpha-j \beta$ where $\beta>0$. Computed results and tangential magnetic field components on a PEC sphere (that has the same radius) are plotted in Figure 3. It is seen by comparing the obtained surface field components with those of a PEC sphere for the limiting $Z_{s}$ value that 

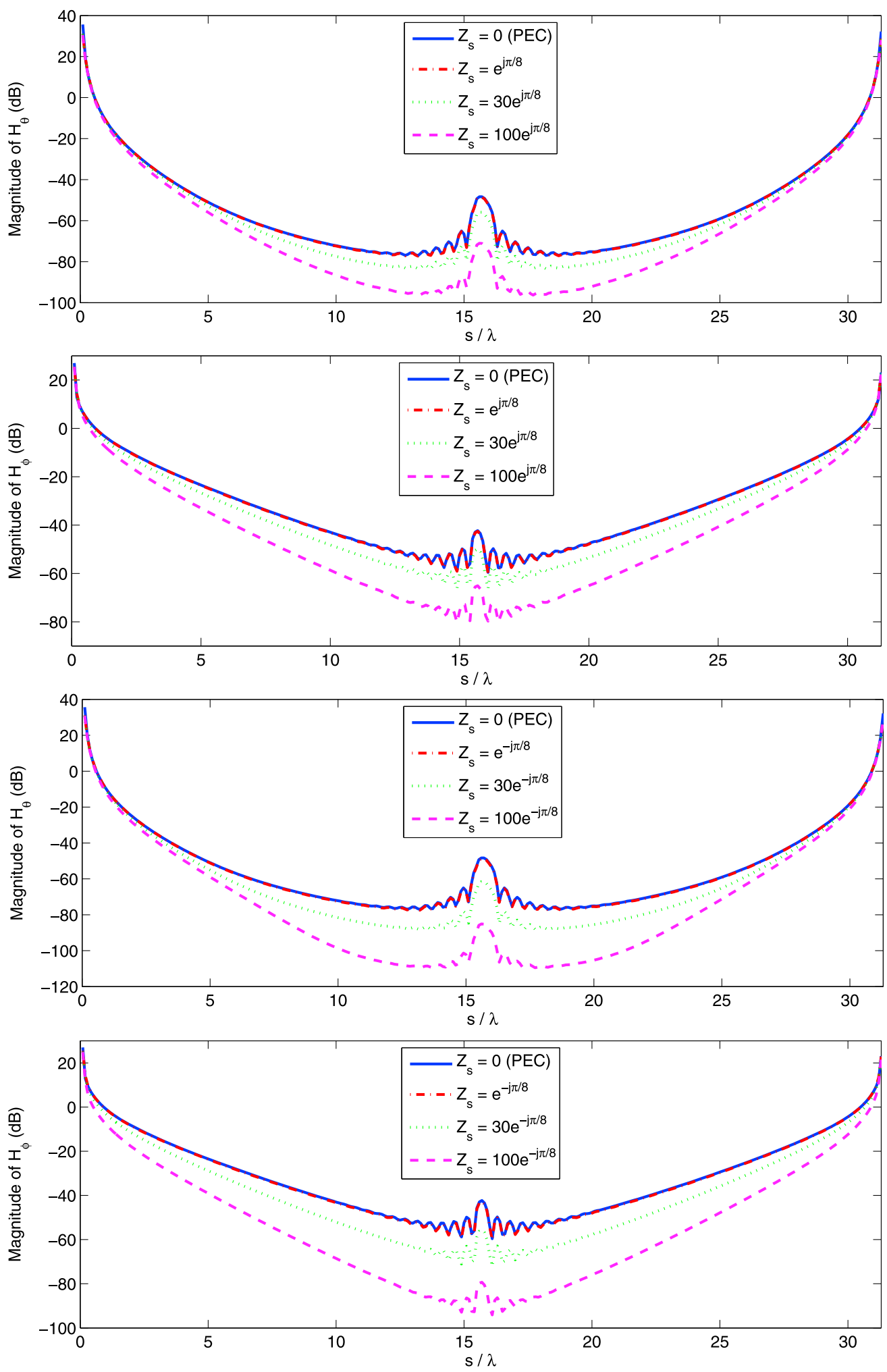

Figure 3

8 of 14 

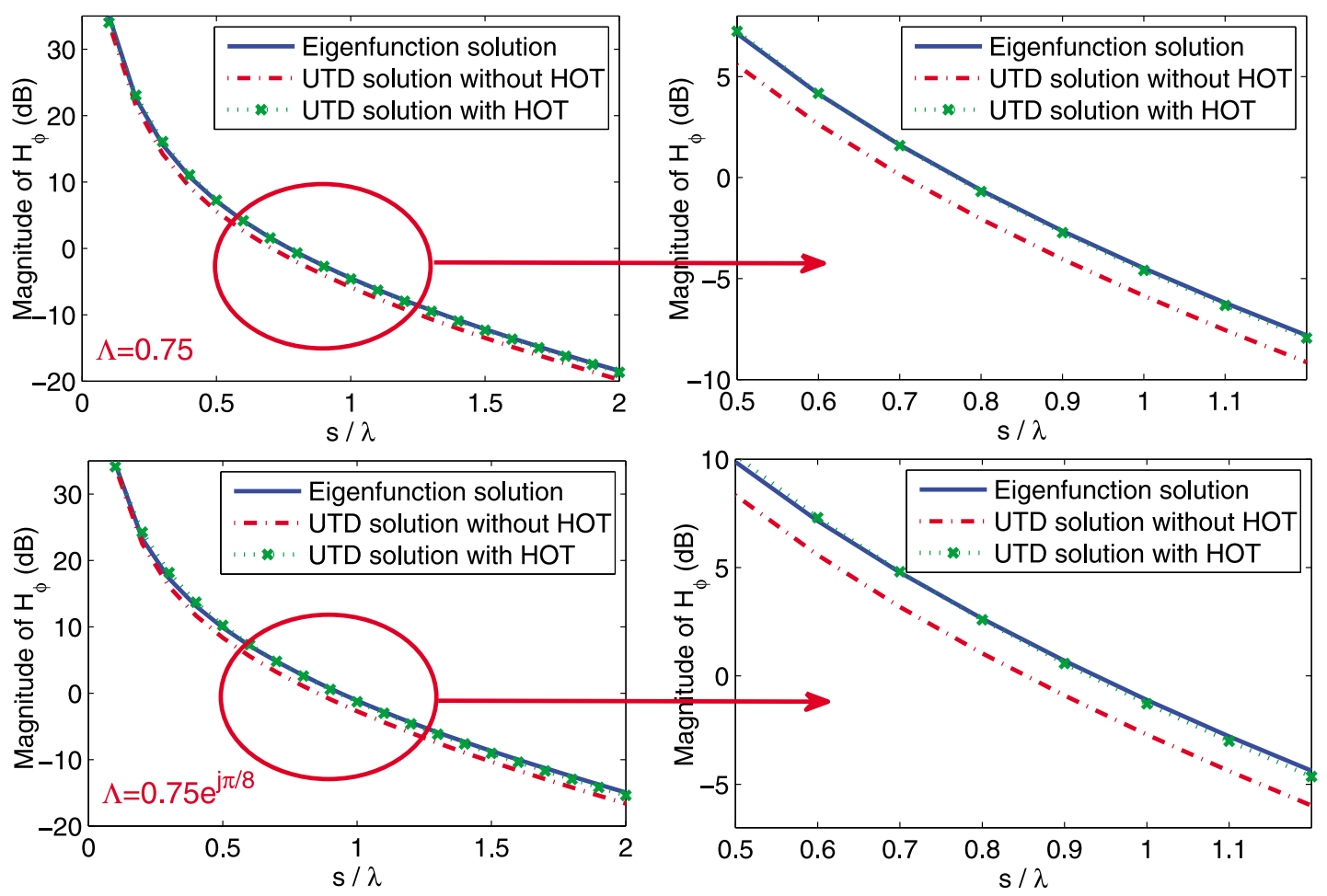

Figure 4. Comparison of the magnitude (in $\mathrm{dB}$ ) of the $H_{\phi}$ component versus separation, $s$, obtained by the eigenfunction solution, the UTD solution with and without the higher-order and derivative terms (referred to as HOT) for an impedance sphere with $a=3 \lambda, \phi=90^{\circ}$ and $\Lambda=0.75$ or $\Lambda=$ $0.75 e^{j \pi / 8}$ at $f=10 G H z$.

when $Z_{s} \rightarrow 0$, impedance sphere results become the PEC sphere results.

[22] The second set of numerical results aims to check (1) the accuracy of the proposed UTD-based solution, and (2) effects of the included higher-order terms and the derivatives of the Fock type integrals. Therefore, the UTD solutions with and without these terms are compared with the eigenfunction solution, in which the fields are obtained without applying high-frequency techniques and involve infinite summations. Note that the infinite summations in the eigenfunction solution may exhibit convergence problems when both the source and observation points are on the surface of the impedance sphere (same is also true for PEC spheres) and the separation, $s$, between them is electrically large. Therefore, although accurate eigenfunction results are obtained for the $H_{\phi}$ component, these summations do not converge for the $H_{\theta}$ component. Because of this reason, the numerical results regarding the $H_{\theta}$ component only compares the two UTD solutions, namely, the one that includes the higher-order terms and the derivatives of the Fock type integrals and the one that does not include them.

[23] Figure 4 shows the $H_{\phi}$ component of the eigenfunction solution and the UTD-based solutions (with and without the higher-order and derivative terms) for the geodesic path length, $s$, varying from $0.1 \lambda$ to $2 \lambda$ at $f=$ $10 \mathrm{GHz}$ for a fixed $\phi=90^{\circ}$ on a sphere with $a=3 \lambda$ and $\Lambda=0.75$ or $\Lambda=0.75 e^{j \pi / 8}$. It is observed that more accurate results are obtained when the higher-order and derivative terms are included. The accuracy in the magnitude improves for about a couple of $\mathrm{dB}$ almost everywhere. It should be mentioned that the higher-order terms in highfrequency solutions usually improve the results for small separations. However, we realized that contributions coming from some of the derivative terms are comparable with the first-order terms. Hence, the accuracy

Figure 3. Comparison of the magnitude (in $\mathrm{dB}$ ) of the $H_{\theta}$ and $H_{\phi}$ versus separation, $s$, obtained by the impedance sphere solution for various $Z_{s}$ values and the PEC sphere solution for $a=5 \lambda, \phi=45^{\circ}$ at $f=7 G H z$. 



Figure 5. Comparison of the magnitude (in $\mathrm{dB}$ ) of the $H_{\theta}$ component versus separation, $s$, obtained by the UTD solution with and without the higher-order and derivative terms (referred to as HOT) for an impedance sphere with $a=3 \lambda, \phi=0^{\circ}$ and $\Lambda=0.75$ or $\Lambda=0.75 e^{j \pi / 8}$ at $f=10 \mathrm{GHz}$.

improves for relatively large $s$ values (see the magnified region where $0.5 \lambda \leq s \leq 1.2 \lambda$ ) as well. Figure 5 shows a similar comparison without the eigenfunction solution for the $H_{\theta}$ component. The sphere parameters are kept the same except $\phi$ is set to $0^{\circ}$. Similar to the $H_{\phi}$ component, 2-3 dB difference in magnitude is visible for almost all $s$ values when the higher-order and derivative terms are included.

[24] Finally, in Figure 6, magnitude and phase of $H_{\phi}$ and $H_{\theta}$ components of the UTD-based solutions with and without higher-order and derivative terms are plotted, and compared with the eigenfunction solution only for $H_{\phi}$ for a fixed $s=1.1 \lambda$ at $f=10 \mathrm{GHz}$ for azimuthal angle varying from $\phi=0^{\circ}$ to $\phi=180^{\circ}$ on a sphere with $a=3 \lambda$ and $\Lambda=1.1 e^{-j \pi / 8}$. Similar to the previous results UTDbased solution with the higher-order and derivative terms yields a better agreement both in magnitude and phase with the eigenfunction solution from those without these terms. The improvement in the magnitude is in the order of $2-5 \mathrm{~dB}$, and the improvement in the phase ranges between $2-3^{\circ}$ to $8-10^{\circ}$. The amount of improvement may vary depending on $Z_{s}, a, \phi$ values. However, usually the variation is small.

\section{Conclusion}

[25] A UTD-based high-frequency asymptotic solution for the surface magnetic field excited by a tangential magnetic source located on the surface of an electrically large sphere with an IBC is presented. The solution contains some higher-order terms and derivatives of Fock type integrals which may become important for some surface impedance values at some regions of the sphere. Accuracy of the proposed solution is assessed by comparing the obtained surface field components with those of a PEC sphere for the limiting $Z_{s}$ value, as well as with the results obtained using an eigenfunction solution. Therefore, the proposed solution is useful in the analysis of mutual coupling between conformal slot/aperture antennas on a thin material coated or partially coated 



Figure 6. Comparison of the magnitude (in $\mathrm{dB}$ ) and phase (in degrees) of the $H_{\phi}$ and $H_{\theta}$ components versus azimuthal angle, $\phi$, obtained by the eigenfunction solution (only for $H_{\phi}$ ), the UTD solution with and without the higher-order and derivative terms (referred to as HOT) for an impedance sphere with $a=3 \lambda, s=1.1 \lambda, \Lambda=1.1 e^{-j \pi / 8}$ at $f=10 G H z$.

sphere. Furthermore, it acts as a canonical problem useful toward the development of asymptotic solutions valid for arbitrary smooth convex thin material coated/partially material coated surfaces.

\section{Appendix A: Approximation of Cylindrical Functions by Fock Type Airy Functions}

[26] The cylindrical functions $\hat{J}_{\mu^{-1 / 2}}, \hat{H}_{\mu^{-1 / 2}}^{(2)}$ and their derivatives can be approximated by the Fock type Airy functions as follows:

$$
\begin{gathered}
\hat{J}_{\mu-1 / 2}(k a) \sim \frac{\sqrt{m}}{2 j}\left[W_{1}(\tau)-W_{2}(\tau)\right] \\
\hat{J}_{\mu-1 / 2}^{\prime}(k a) \sim-\frac{1}{2 j \sqrt{m}}\left[W_{1}^{\prime}(\tau)-W_{2}^{\prime}(\tau)\right] \\
\hat{H}_{\mu-1 / 2}^{(2)}(k a) \sim j \sqrt{m} W_{2}(\tau)
\end{gathered}
$$

$$
\hat{H}_{\mu-1 / 2}^{(2)^{\prime}}(k a) \sim \frac{1}{j \sqrt{m}} W_{2}^{\prime}(\tau)
$$

where [Fock, 1965]

$$
\begin{aligned}
& W_{1}(\tau)=\frac{1}{\sqrt{\pi}} \int_{\infty e^{-j 2 \pi / 3}}^{\infty} e^{\tau t-t^{3} / 3} d t \\
& W_{2}(\tau)=\frac{1}{\sqrt{\pi}} \int_{\infty e^{j 2 \pi / 3}}^{\infty} e^{\tau t-t^{3} / 3} d t .
\end{aligned}
$$

\section{Appendix B: Obtaining Field Expressions From Potentials}

[27] Recall that the high-frequency based expressions for the potentials, $\psi^{e}$ and $\psi^{m}$, are provided in (33) and (39), respectively, as

$$
\psi_{ \pm}^{e} \sim \pm \frac{j p_{m}}{k} V_{z}\left(\xi^{ \pm}\right) \sin \phi D^{ \pm} G\left(k s^{ \pm}\right)
$$




$$
\begin{aligned}
\psi_{ \pm}^{m} \sim & \pm \frac{-p_{m} e^{-j \pi / 4}}{4 k m Y_{0}} \cos \phi D^{ \pm} G\left(k s^{ \pm}\right) \sqrt{\frac{\xi^{ \pm}}{\pi}} \int_{-\infty}^{\infty} \\
& \cdot d \tau e^{-j \xi^{ \pm} \tau} \frac{W_{2}^{\prime}(\tau)}{-m W_{2}(\tau)+j \Lambda W_{2}^{\prime}(\tau)} \\
& \cdot\left\{W_{1}\left(\tau-y_{2}\right)\left[-m W_{2}(\tau)+j \Lambda W_{2}^{\prime}(\tau)\right]\right. \\
& \left.+W_{2}\left(\tau-y_{2}\right)\left[m W_{1}(\tau)-j \Lambda W_{1}^{\prime}(\tau)\right]\right\}
\end{aligned}
$$

where $G\left(k s^{ \pm}\right), D^{ \pm}$, and $V_{z}\left(\xi^{ \pm}\right)$are given in (34)-(36). Then, the derivative of $\psi^{m}$ with respect to $r$ is evaluated as follows ( \pm is dropped for convenience):

$$
\begin{aligned}
\left.\frac{\partial}{\partial r} \psi^{m}\right|_{r=a}= & \frac{-p_{m} e^{-j \pi / 4}}{4 k m Y_{0}} \cos \phi D G(k s) \sqrt{\frac{\xi}{\pi}}\left(\frac{-k}{m}\right) \int_{-\infty}^{\infty} \\
& \cdot d \tau e^{-j \xi \tau} \frac{W_{2}^{\prime}(\tau)}{-m W_{2}(\tau)+j \Lambda W_{2}^{\prime}(\tau)} \\
& \cdot\left\{W_{1}^{\prime}(\tau)\left[-m W_{2}(\tau)+j \Lambda W_{2}^{\prime}(\tau)\right]\right. \\
& \left.+W_{2}^{\prime}(\tau)\left[m W_{1}(\tau)-j \Lambda W_{1}^{\prime}(\tau)\right]\right\} \\
= & \frac{-p_{m} e^{-j \pi / 4}}{2 m^{2} Y_{0}} \cos \phi D G(k s) \sqrt{\frac{\xi}{\pi}} \int_{-\infty}^{\infty} \\
& \cdot d \tau e^{-j \xi \tau} \frac{j m W_{2}^{\prime}(\tau)}{-m W_{2}(\tau)+j \Lambda W_{2}^{\prime}(\tau)} \\
= & \frac{-j p_{m}}{2 \xi m^{2} Y_{0}} \cos \phi D G(k s) e^{j 3 \pi / 4} \frac{\xi^{3 / 2}}{\sqrt{\pi}} \int_{-\infty}^{\infty} \\
& \cdot d \tau e^{-j \xi \tau} \frac{-R_{w}(\tau) q_{m}}{R_{w}(\tau)-q_{m}} \\
= & \frac{-j p_{m}}{2 \xi m^{2} Y_{0}} U_{z} \xi \cos \phi D G(k s)
\end{aligned}
$$

where

$$
U_{z}(\xi)=e^{j 3 \pi / 4} \xi^{3 / 2} \frac{1}{\sqrt{\pi}} \int_{-\infty}^{\infty} d \tau e^{-j \xi \tau} \frac{-R_{w} q_{m}}{\left(R_{w}-q_{m}\right)}
$$

in which $q_{m}=-j m \Lambda^{-1}$. On the other hand, the derivative of $G(k s)$ with respect to $\theta$ is given by

$$
\begin{aligned}
\frac{\partial}{\partial \theta} G(k s) & =\frac{k^{2} Y_{0}}{2 \pi j} \frac{\partial}{\partial \theta} \frac{e^{-j k s}}{k s}=\frac{k^{2} Y_{0}}{2 \pi j} a \frac{\partial}{\partial s} \frac{e^{-j k s}}{k s} \\
& =k a\left(-j-\frac{1}{k s}\right) \frac{k^{2} Y_{0}}{2 \pi j} \frac{e^{-j k s}}{k s} \\
& =2 m^{3}\left(-j-\frac{1}{k s}\right) G(k s)
\end{aligned}
$$

and using (15) and (37), the final expressions for tangential magnetic field components, $H_{\theta}$ and $H_{\phi}$, are found as

$$
\begin{aligned}
H_{\theta}= & \frac{1}{a \sin \theta} \frac{\partial}{\partial \phi}\left(\frac{j p_{m}}{k} V_{z}(\xi) \sin \phi D G(k s)\right) \\
& +\frac{1}{j k Z_{0} a} \frac{\partial}{\partial \theta}\left(\frac{-j p_{m}}{2 \xi m^{2} Y_{0}} U_{z}(\xi) \cos \phi D G(k s)\right) \\
= & \frac{j p_{m}}{k a \sin \theta} V_{z}(\xi) \cos \phi D G(k s)-\frac{p_{m} \cos \phi}{2 k a m^{2}} \\
& \cdot\left(\frac{1}{\xi} D U_{z}(\xi) \frac{\partial G(k s)}{\partial \theta}+D U_{z}(\xi) G(k s)\right. \\
& \left.\cdot \frac{\partial}{\partial \theta}\left(\frac{1}{\xi}\right)+G(k s) \frac{1}{\xi} \frac{\partial}{\partial \theta}\left[D U_{z}(\xi)\right]\right) \\
= & p_{m} \cos \phi \frac{j}{k s} V_{z}(\xi) D^{3} G(k s)-\frac{p_{m} \cos \phi}{2 k a m^{2}} \\
& \cdot\left(D G(k s) U_{z}(\xi) \frac{2 m^{3}}{\xi}\left(-j-\frac{1}{k s}\right)+D G(k s) U_{z}(\xi)\right. \\
& \left.\cdot \frac{-1}{\xi \theta}+G(k s) \frac{1}{\xi} \frac{\partial}{\partial \theta}\left[D U_{z}(\xi)\right]\right) \\
= & p_{m} \cos \phi\left\{\left[\frac{j}{k s} V_{z}(\xi) D^{2}+U_{z}(\xi) \frac{j}{k s}\left(1-\frac{2 j}{k s}\right)\right]\right. \\
& \left.\cdot D G(k s)-G(k s) \frac{1}{4 m^{5} \xi} \frac{\partial}{\partial \theta}\left[D U_{z}(\xi)\right]\right\} \\
& \left.+G(k s) \frac{j}{2 m^{3}} \frac{\partial}{\partial \theta}\left[D V_{z}(\xi)\right]\right\} . \\
& +\frac{p_{m} \sin \phi}{2 k a \sin \theta \xi m^{2}} G(k s) \frac{1}{\xi} D U_{z} \\
H_{\phi}= & -\frac{1}{a} \frac{\partial}{\partial \theta}\left(\frac{j p_{m}}{k} V_{z}(\xi) \sin \phi D G(k s)\right)+\frac{1}{j k Z_{0} a \sin \theta} \frac{\partial}{\partial \phi} \sin \phi\left\{\left[1-\frac{j}{k s}\right) V_{z}(\xi)+j^{2} D^{2} \frac{U_{z}(\xi)}{(k s)^{2}}\right] D G(k s) \\
& \cdot\left(\frac{-j p_{m}}{2 \xi m^{2} Y_{0}} U_{z}(\xi) \cos \phi D G(k s)\right) \\
= & \frac{j p_{m}}{k a} \sin \phi\left(V_{z}(\xi) D \frac{\partial G(k s)}{\partial \theta}+G(k s) \frac{\partial}{\partial \theta}\left[D V_{z}(\xi)\right]\right) \\
& (\mathrm{B} 7) \\
&
\end{aligned}
$$

[28] Making the use of (B6) together with the following expressions

$$
\frac{\partial}{\partial \theta} D=\frac{m^{3}}{k s} D\left(1-D^{2} \cos \theta\right),
$$




$$
\begin{gathered}
\frac{\partial}{\partial \theta} U_{z}(\xi)=\frac{3 m}{2 \xi} U_{z}(\xi)+e^{j 3 \pi / 4} \xi^{3 / 2} \frac{1}{\sqrt{\pi}} \int_{-\infty}^{\infty} \\
\cdot d \tau(-j m \tau) e^{-j \xi \tau} \frac{-R_{w} q_{m}}{\left(R_{w}-q_{m}\right)}, \\
\tilde{U}_{z}(\xi)=e^{j 3 \pi / 4} \xi^{3 / 2} \frac{1}{\sqrt{\pi}} \int_{-\infty}^{\infty} d \tau e^{-j \xi \tau} \frac{-R_{w} q_{m}}{\left(R_{w}-q_{m}\right)}\left(1+\frac{\tau}{2 m^{2}}\right),
\end{gathered}
$$

$H_{\theta}$ can be written as

$$
\begin{aligned}
H_{\theta}= & p_{m} \cos \phi\left\{\left[\frac{j}{k s} V_{z}(\xi) D^{2}+U_{z}(\xi) \frac{j}{k s}\left(1-\frac{2 j}{k s}\right)\right]\right. \\
& \cdot D G(k s)-G(k s) D U_{z}(\xi)\left[\frac{\left(1-D^{2} \cos \theta\right)}{4 m^{2} k s \xi}+\frac{3}{8 m^{4} \xi^{2}}\right] \\
& -\frac{G(k s) D}{4 m^{5} \xi} e^{j 3 \pi / 4} \xi^{3 / 2} \frac{1}{\sqrt{\pi}} \int_{-\infty}^{\infty} \\
& \left.\cdot d \tau(-j m \tau) e^{-j \xi \tau} \frac{-R_{w} q_{m}}{\left(R_{w}-q_{m}\right)}\right\} \\
= & p_{m} \cos \phi\left[\frac{j}{k s} V_{z}(\xi) D^{2}+U_{z}(\xi) \frac{j}{k s}\left(1-\frac{2 j}{k s}\right)\right. \\
& +U_{z}(\xi)\left(\frac{D^{2} \cos \theta}{2(k s)^{2}}-\frac{2}{(k s)^{2}}\right)+\frac{j}{k s} e^{j 3 \pi / 4} \xi^{3 / 2} \frac{1}{\sqrt{\pi}} \int_{-\infty}^{\infty} \\
& \left.\cdot d \tau \frac{\tau}{2 m^{2}} e^{-j \xi \tau} \frac{-R_{w} q_{m}}{\left(R_{w}-q_{m}\right)}\right] D G(k s) \\
= & p_{m} \cos \phi\left[\left(\frac{j}{k s}\right) \tilde{U}_{z}(\xi)-\frac{D^{2}}{2}\left(\frac{j}{k s}\right)^{2}\right. \\
& \left.\cdot \cos \theta U_{z}(\xi)+D^{2} \frac{j}{k s^{ \pm}} V_{z}(\xi)\right] D G(k s) .
\end{aligned}
$$

[29] Similarly, using (B7), (B8) together with the following expressions

$$
\begin{gathered}
\frac{\partial}{\partial \theta} V_{z}(\xi)=\frac{m^{3}}{k s} V_{z}(\xi)+\sqrt{\frac{j \xi}{4 \pi}} \int_{-\infty}^{\infty} \frac{1}{R_{w}-q_{e}} e^{-j \xi \tau}(-j m \tau) d \tau \\
\tilde{V}_{z}(\xi)=\sqrt{\frac{j \xi}{4 \pi}} \int_{-\infty}^{\infty} d \tau e^{-j \xi \tau} \frac{1}{\left(R_{w}-q_{e}\right)}\left(1+\frac{\tau}{2 m^{2}}\right)
\end{gathered}
$$

$H_{\phi}$ can be written as

$$
\begin{aligned}
H_{\phi}= & -p_{m} \sin \phi\left\{\left[\left(1-\frac{j}{k s}\right) V_{z}(\xi)+j^{2} D^{2} \frac{U_{z}(\xi)}{(k s)^{2}}\right] D G(k s)\right. \\
& +G(k s) D V_{z}(\xi) \frac{j}{2 m^{3}}\left[\frac{m^{3}}{k s}\left(1-D^{2} \cos \theta\right)+\frac{m^{3}}{k s}\right] \\
& \left.+\frac{j G(k s) D}{2 m^{3}} \sqrt{\frac{j \xi}{4 \pi}} \int_{-\infty}^{\infty} d \tau e^{-j \xi \tau} \frac{1}{\left(R_{w}-q_{e}\right)}\left(1+\frac{\tau}{2 m^{2}}\right)\right\} \\
= & -p_{m} \sin \phi\left[\left(1-\frac{j}{k s}\right) V_{z}(\xi)+j^{2} D^{2} \frac{U_{z}(\xi)}{(k s)^{2}}\right. \\
& +V_{z}(\xi)\left(\frac{j}{2 k s}\left(1-D^{2} \cos \theta\right)+\frac{j}{2 k s}\right) \\
& \left.+\sqrt{\frac{j \xi}{4 \pi}} \int_{-\infty}^{\infty} \frac{1}{R_{w}-q_{e}} e^{-j \xi \tau} \frac{\tau}{2 m^{2}} d \tau\right] D G(k s) \\
= & p_{m} \sin \phi\left[\tilde{V}_{z}(\xi)-\frac{D^{2}}{2} \frac{j}{k s} \cos \theta V_{z}(\xi)+D^{2}\left(\frac{j}{k s}\right)^{2} U_{z}(\xi)\right] \\
& \cdot D G(k s) .
\end{aligned}
$$

\section{References}

Abramowitz, M., and I. A. Stegun (1964), Handbook of Mathematical Functions With Formulas, Graphs, and Mathematical Tables, Dover, New York.

Alisan, B., V. B. Ertürk, and A. Altintas (2006), Efficient computation of nonparaxial surface fields excited on an electrically large circular cylinder with an impedance boundary condition, IEEE Trans. Antennas Propag., 54(9), 2559-2567.

Balanis, C. A. (1989), Advanced Engineering Electromagnetics, John Wiley, New York.

Bremmer, H. (1958), Applications of operational calculus to ground-wave propagation, particularly for long waves, IRE Trans. Antennas Propag., 6, 267-272.

Felsen, L. B., and K. Naishadham (1991), Ray formulation of waves guided by circular cylindrical stratified dielectrics, Radio Sci., 26, 203-209.

Fock, V. A. (1945), Diffraction of radio waves around the earth's surface, J. Phys. (Moscow), 9, 256-266.

Fock, V. A. (1965), Electromagnetic Diffraction and Propagation Problems, Pergamon, New York.

Harrington, R. F. (1961), Time-Harmonic Electromagnetic Fields, IEEE Press, New York.

Hill, D. A., and J. R. Wait (1980), Ground wave attenuation function for a spherical earth with arbitrary surface impedance, Radio Sci., 15(3), 637-643. 
Hill, D. A., and J. R. Wait (1981), HF ground wave propagation over sea ice for a spherical earth model, IEEE Trans. Antennas Propag., 29(3), 525-527.

King, R. J., and J. R. Wait (1976), Electromagnetic ground wave propagation, in Theory and Experiment, Symposia Mathematica, vol. 18, pp. 107-208, Academic, New York.

Kouyoumjian, R. G., and P. H. Pathak (1974), A uniform geometrical theory of diffraction for an edge in a perfectly conducting surface, Proc. IEEE, 62, 1448-1461.

Logan, N. A., and K. S. Yee (1962), A simple expression for propagation constants associated with a reactive convex surface, IRE Trans. Antennas Propag., 10, 103.

Pathak, P. H., and N. Wang (1978), An analysis of mutual coupling between antennas on a smooth convex surface, Rep. 784583-7, ElectroSci. Lab., Dep. of Electr. Eng., Ohio State Univ., Columbus.

Pathak, P. H., and N. Wang (1981), Ray analysis of mutual coupling between antennas on a convex surface, IEEE Trans. Antennas Propag., 29, 911-922.

Penney, C. W., R. J. Luebbers, and J. W. Schuster (1996), Scattering from coated targets using frequency-dependent, surface impedance boundary condition in FDTD, IEEE Trans. Antennas Propag., 44, 434-443.

Rojas, R. G., and Z. Al-hekail (1989), Generalized impedance/ resistive boundary conditions for electromagnetic scattering problems, Radio Sci., 24, 1-12.

Senior, T. B. A., and J. L. Volakis (1995), Approximate Boundary Conditions in Electromagnetics, IEEE Press, London.

Sipus, Z., S. Skokic, M. Bosiljevac, and N. Burum (2008), Study of mutual coupling between circular stacked-patch antennas on a sphere, IEEE Trans. Antennas Propag., 56, 1834-1844.

Spies, K., and J. R. Wait (1966), On the calculation of the ground wave attenuation factor at low frequencies, IEEE Trans. Antennas Propag., 14, 515-517.
Spies, K. P., and J. R. Wait (1967), On the calculation of antenna patterns for an inhomogeneous spherical earth, Radio Sci., 2(11), 1361-1378.

Tokgöz, C., and R. J. Marhefka (2006), A UTD based asymptotic solution for the surface magnetic field on a source excited circular cylinder with an impedance boundary condition, IEEE Trans. Antennas Propag., 54(6), 1750-1757.

Tomasic, B., J. Turtle, and S. Liu (2002), A geodesic sphere phased array for satellite control and communication, paper presented at the XXVIIth General Assembly, Int. Union of Radio Sci., Maastricht, Netherlands.

Wait, J. R. (1956), Radiation from a vertical antenna over a curved stratified ground, Natl. Bur. Stand. J. Res., 56, 237-244.

Wait, J. R. (1958), On the theory of propagation of electromagnetic waves along a curved surface, Can. J. Phys., 36, 9-17.

Wait, J. R. (1960), On the excitation of electromagnetic surface waves on a curved surface, IRE Trans. Antennas Propag., 8 , 445-449.

Wait, J. R. (1962), The propagation of EM waves along the earth's surfaces, in Electromagnetic Waves, edited by R. E. Langer, pp. 243-290, Univ. of Wis. Press, Madison.

Wait, J. R. (1965), A note on surface waves and ground waves, IEEE Trans. Antennas Propag., 13, 996-997.

Wait, J. R. (1967), Illumination of an inhomogeneous spherical earth by an LF plane electromagnetic wave, Radio Sci., 2(1), 111-118.

Wait, J. R. (1998), The ancient and modern history of EM ground wave propagation, IEEE Trans. Antennas Propag. Mag., 40(5), 7-24.

Watson, G. N. (1918), The diffraction of electric waves by the earth, Proc. R. Soc. A, 95, 83-99.

B. Alisan and V. B. Ertürk, Department of Electrical and Electronics Engineering, Bilkent University, TR-06800 Ankara, Turkey. (alisan@ee.bilkent.edu.tr; vakur@ee.bilkent.edu.tr) 